\title{
Gerontology
}

\section{Does a Single Session of High-Intensity Interval Training Provoke a Transient Elevated Risk of Falling in Seniors and Adults?}

\author{
Lars Donath $^{\mathrm{a}}$ Eduard Kurz $^{\mathrm{b}}$ Ralf Roth $^{\mathrm{a}}$ Henner Hanssen ${ }^{\mathrm{a}}$ \\ Arno Schmidt-Trucksäss $^{a}$ Lukas Zahner ${ }^{a}$ Oliver Faude ${ }^{a}$ \\ ${ }^{a}$ Department of Sport, Exercise and Health, University of Basel, Basel, Switzerland; ${ }^{b}$ Clinic for Trauma, \\ Hand and Reconstructive Surgery, Division of Motor Research, Pathophysiology and Biomechanics, \\ Jena University Hospital, Jena, Germany
}

\section{Key Words}

Upright stance $\cdot$ Postural control $\cdot$ Elderly $\cdot$ Exercise

training $\cdot$ Balance $\cdot$ Risk of falling

\begin{abstract}
Background: Balance and strength training can reduce seniors' fall risk up to $50 \%$. Available evidence suggests that acute bouts of neuromuscular and endurance exercise deteriorate postural control. High-intensity endurance training has been successfully applied in different populations. Thus, it seemed valuable to examine the acute effects of high-intensity interval training (HIIT) on neuromuscular performance in seniors and young adults. Objective: The acute impact of a HIIT session on balance performance and muscle activity after exercise cessation and during post-exercise recovery was examined in young and old adults. We intended to investigate whether a transient exercise-induced fall-risk may occur in both groups. Methods: 20 healthy seniors (age 70 (SD 4) years) and young adults (age 27 (SD 3) years) were examined on 3 days. After exhaustive ramp-like treadmill testing in order to determine maximal heart rate $\left(\mathrm{HR}_{\max }\right)$ on the first day, either a $4 \times 4$ min HIIT at $90 \%$ of $\mathrm{HR}_{\max }$ or a control condition (CON) was randomly performed on the second
\end{abstract}

and third day, respectively. Balance performance (postural sway) was assessed during single limb stance with open eyes (SLEO) and double limb stance with closed eyes (DLEC). EMG was recorded for the soleus (SOL), anterior tibialis (TIB), gastrocnemius (GM) and peroneus longus $(\mathrm{PL})$ muscles at the dominant leg. All measures were collected before, immediately as well as 10, 30 and 45 min after HIIT and CON, respectively. Results: Compared to CON, HIIT induced significant increases of postural sway immediately after exercise cessation during SLEO in both groups (adults: $p<0.001, \Delta=+25 \%$ sway; seniors: $p=0.007, \Delta=+15 \%$ sway). Increased sway during DLEC was only found for seniors immediately and 10 min after HIIT (post: $\mathrm{p}=0.003, \Delta=+14 \%$ sway, 10 min post: $p=0.004, \Delta=+18 \%$ sway). Muscle activity was increased during SLEO for TIB until 10 min post in seniors $(0.008<p<0.03)$ and immediately after HIIT in adults $(p<0.001)$. Conclusion: HIIT training may cause an acute 'open-fall-window' with a transient impairment of balance performance for at least 10 min after exercise cessation in both groups. Occluded vision in seniors seems to prolong this period up to $30 \mathrm{~min}$. Thus, the advantage of HIIT with regard to time efficiency seems debatable when considering transient HIIT-induced impairments of neuromuscular function.

(c) 2014 S. Karger AG, Basel

\section{KARGER 125}

(c) 2014 S. Karger AG, Basel

0304-324X/14/0611-0015\$39.50/0

E-Mail karger@karger.com

www.karger.com/ger
Lars Donath

Department of Sport, Exercise and Health

University of Basel, Birsstrasse 320B

$\mathrm{CH}-4052$ Basel (Switzerland)

E-Mail lars.donath@unibas.ch 


\section{Introduction}

Seniors aged $\geq 65$ years of age are expected to constitute nearly $30 \%$ of the societal proportion in Western countries until the end of the current century [1]. This age-group remarkably affects healthcare utilization and expenditures [2]. In this regard, fall-related injuries are considered a major economic, public and individual health concern. Reported annual fall rates range between 0.3 and 1.6 falls (weighted mean 0.6 ) per person in this age group [3]. The estimated healthcare costs amount to USD 0.2 billion for fatal and USD 19 billion for non-fatal fall-related injuries [4].

Extrinsic (e.g. missing handrail, uneven terrain, twilight, obstacles) and intrinsic factors (e.g. strength deficits of the lower limbs, increased gait variability, impaired balance performance) were reported to increase the risk of falling. In turn, strength and balance training have been shown to reduce the risk of falling up to $50 \%$ by improving intrinsic risk factors (maximal strength, explosive power, static and dynamic balance, gait performance) in older adults [5-8]. There is also evidence that acute bouts of exercise may relevantly deteriorate static and dynamic balance performance. Most of these studies, however, reported fatigue-induced alterations of balance and gait performance after repetitive sit-to-stand tasks and local lower-extremity or trunk muscle resistance exercises in seniors [9-12]. Only two studies investigated acute effects of submaximal and maximal cycling or walking on balance performance in seniors $[13,14]$. Donath et al. [14] observed increased postural sway after an exhaustive treadmill walking test but not after a submaximal $2-\mathrm{km}$ walking test. Stemplewski et al. [13] also found decreased postural control after $10 \mathrm{~min}$ of submaximal cycling at $60 \%$ of heart rate reserve. However, both studies did not measure exercise-induced changes of muscle activity and the time course of balance recovery after exercise cessation. Furthermore, neither study analyzed changes of balance performance after acute exercise bouts in a group of young adults and seniors.

As high-intensity interval training (HIIT) has been shown to improve maximal oxygen uptake and cardiac capacity (stroke volume) up to $15 \%$ in young adults [15], HIIT has been successfully applied in clinical and older population [16-18]. Compared to continuous submaximal aerobic exercise training, HIIT thus seems to serve as a feasible and time-efficient training regimen also for seniors.

In corroboration with the above-mentioned studies on acute exercise effects on neuromuscular performance, the present study aimed at investigating the impact of a typical HIIT session on standing balance performance, muscle activity and balance recovery in young adults and seniors as well. We assumed that HIIT $(4 \times 4$ min at 90 $95 \% \mathrm{HR}_{\max }$ ) leads to alterations of standing balance and muscle activity in both groups only during demanding standing balance tasks.

\section{Methods}

Study Design

On the basis of the physical activity readiness questionnaire (PAR-Q) and a resting ECG in supine position (Custo cardio 100; Custo med $\mathrm{GmbH}$, Ottobrunn, Germany), all participants were initially examined by an experienced physician. Exercise ECG and maximal heart rate $\left(\mathrm{HR}_{\max }\right)$ were assessed in all participants on the first day via exhaustive ramp-like treadmill exercise testing. The order of the subsequent two testing conditions (HIIT and control condition (CON)) was randomly assigned for each individual. Hence, the present study was designed as a randomized controlled cross-over study (fig. 1). The testing days were interspersed with a break between 48 and $72 \mathrm{~h}$. All measurements were performed at a similar time of day for each individual. The Freiburg Physical Activity Questionnaire was used to assess physical activity data [19]. Balance and muscle activity data were examined before and immediately after the HIIT session. To additionally obtain postexercise recovery data, 10, 30 and $45 \mathrm{~min}$ after HIIT cessation, balance and muscle activity were recorded. The study was approved by the local ethics committee (Ethikkommission beider Basel (EKBB), Basel, Switzerland, 334/12) and complied with the Declaration of Helsinki. All participants signed an informed written consent prior to the start of the study.

\section{Participants}

Twenty healthy, active seniors older than 65 years of age and 20 young, active adults were enrolled in the present study (table 1). No participant reported medication intake or health impairments that may adversely affect balance, maximal exercise or muscle activity testing. Seniors who reported diabetes, untreated hypertension (>180/110 mm Hg), glaucoma, endoprosthesis, arthritis and arthrosis, heart failure, coronary heart disease, stroke, obstructive diseases, eczema and $\beta$-blocker users were not included in the study. All participants were asked to refrain from strenuous exercise $72 \mathrm{~h}$ prior to the exercise tests.

\section{Acute Interventions}

Maximal Ramp Exercise. In order to determine $\mathrm{HR}_{\max }$ and awkward separation of oxygen uptake $\left(\mathrm{VO}_{2 \max }\right)$, an exhaustive ramp-like exercise test on a treadmill was performed (h/p Cosmos, Pulsar 4.0; HP-Cosmos Sports \& Medical GmbH, Germany). Heart rate (RS 400; Polar Electro Oy, Kempele, Finland) and gas exchange data (Metalyzer 3B; Cortex Biophysik GmbH, Leipzig, Germany) were continuously collected. The age-adapted walkingbased 'Pepper protocol' was applied to the seniors [20]. This exercise protocol started with an inclination of $0 \%$ and a velocity of 1.5 $\mathrm{mph}(2.4 \mathrm{~km} / \mathrm{h})$. Exercise intensity was increased every minute by elevating either treadmill inclination or velocity. A modified running-based Bruce protocol until objective exhaustion with velocity
16

Gerontology 2015;61:15-23 DOI: $10.1159 / 000363767$
Donath/Kurz/Roth/Hanssen/ Schmidt-Trucksäss/Zahner/Faude 


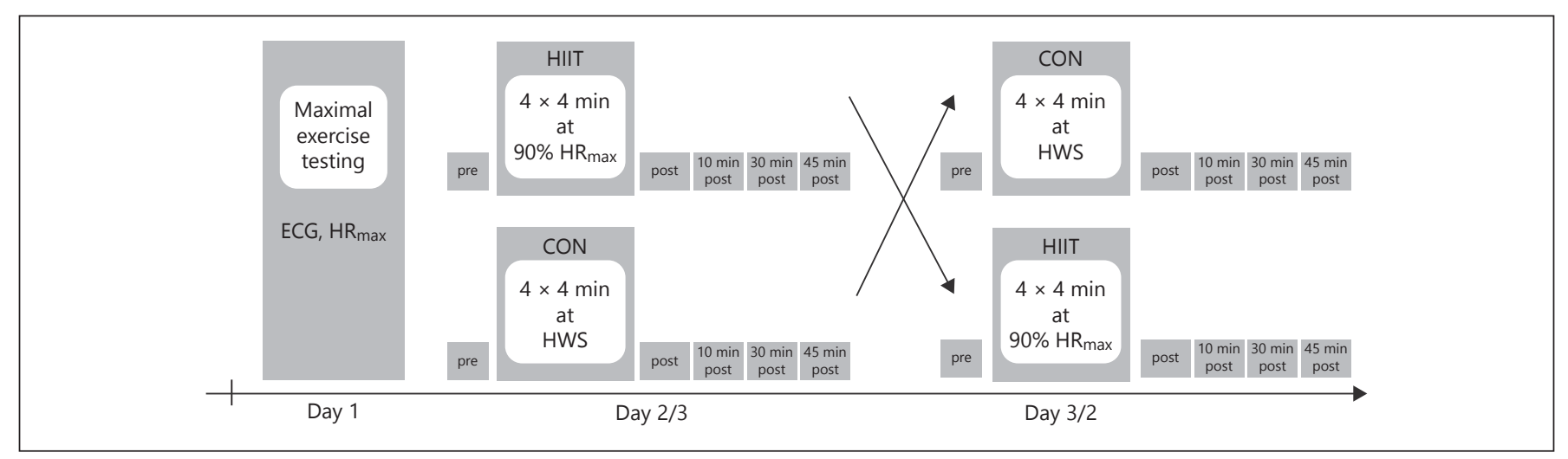

Fig. 1. Randomized crossover study design. The first day was set as a ramp exercise test in order to examine $\mathrm{HR}_{\max }$ and to obtain an exercise ECG. On the second and third day, respectively, either an acute bout of HIIT or a CON condition at normal, habitual walking speed (HWS) was randomly performed.

Table 1. Anthropometric and maximal exercise data of the participants (mean \pm SD values)

\begin{tabular}{|c|c|c|}
\hline & $\begin{array}{l}\text { Young adults } \\
(\mathrm{n}=20)\end{array}$ & $\begin{array}{l}\text { Seniors } \\
(n=20)\end{array}$ \\
\hline \multicolumn{3}{|l|}{ Personal data } \\
\hline Female/male & $9 / 11$ & $12 / 8$ \\
\hline Age, years & $27.1 \pm 3.0$ & $70.0 \pm 3.8$ \\
\hline Weight, kg & $70.5 \pm 11.6$ & $71.1 \pm 12.0$ \\
\hline Height, m & $1.77 \pm 0.09$ & $1.68 \pm 0.09$ \\
\hline Body mass index, $\mathrm{kg} / \mathrm{m}^{2}$ & $22.4 \pm 2.2$ & $25.0 \pm 3.6$ \\
\hline Body fat, $\%$ & $17.9 \pm 5.9$ & $27.0 \pm 9.1$ \\
\hline Physical activity, h.week ${ }^{-1}$ & $8.7 \pm 4.1$ & $10.9 \pm 5.8$ \\
\hline \multicolumn{3}{|l|}{ Maximal ramp test } \\
\hline $\mathrm{T}_{\max }, \min$ & $16.6 \pm 3.5$ & $16.8 \pm 3.8$ \\
\hline $\mathrm{VO}_{2 \max }, 1 \cdot \min ^{-1} \cdot \mathrm{kg}^{-1}$ & $46.6 \pm 7.2$ & $33.2 \pm 6.1$ \\
\hline $\mathrm{HR}, \min ^{-1}$ & $195 \pm 5$ & $161 \pm 13$ \\
\hline Predicted HR, $\mathrm{min}^{-1 \mathrm{a}}$ & $189 \pm 2$ & $159 \pm 3$ \\
\hline $\mathrm{BR}, \mathrm{min}^{-1}$ & $53.4 \pm 5.0$ & $42.2 \pm 6.6$ \\
\hline RER & $1.16 \pm 0.07$ & $1.19 \pm 0.10$ \\
\hline Borg & $9.4 \pm 0.8$ & $8.9 \pm 0.8$ \\
\hline
\end{tabular}

Physical activity is derived from the Freiburger Physical Activity Questionnaire; $\mathrm{T}_{\max }=$ exercise time during the maximal ramplike exercise test; Borg = subjectively perceived exertion level at the Borg CR-10 scale; Borg $_{\text {sub }}=$ averaged perceived exertion level during the $2-\mathrm{km}$ walking test; $\mathrm{BR}_{\max }=$ breathing rate . [40].

${ }^{\text {a }}$ Age-predicted maximal heart rate according to Tanaka et al.

increases of $0.5 \mathrm{~km} / \mathrm{h}$ per minute at a constant inclination of $1 \%$ was applied to the young adults. Thereby, starting velocity was set between 8 and $11 \mathrm{~km} / \mathrm{h}$ depending on the individual state of physical fitness (based on subjective investigator assessment). Exhaustion was assumed when 3 out of 4 objective criteria were achieved:
The remaining testing condition was conducted on day 3. During the second and third day, balance performance and muscle activity was measured before (pre) the $4 \times 4$ interventions as well as immediately (post), 10, 30 and 45 min after exercise cessation.

heart rate above the age-predicted maximum $(208-0.7 \times$ age $)$, ventilatory equivalent for oxygen uptake $\left(\mathrm{VE} / \mathrm{VO}_{2}\right)>35$, respiratory exchange ratio (RER) $>1.15$, and breathing rate $(\mathrm{BR})>40[21]$.

High-Intensity Interval Session. Four consecutive high-intensity intervals of 4 min duration at a target exercise intensity of 90 of $\mathrm{HR}_{\max }$ were completed on a treadmill. These intervals were interspersed with active rest periods of $3 \mathrm{~min}$ at $70 \% \mathrm{HR}_{\max }$ [15]. In order to stay in-between the required heart rate range, treadmill inclination and velocity were adjusted if necessary. Seniors walked briskly with inclination to avoid locomotor or coordinative limitations. The young adults were allowed to run. Heart rate and gas exchange data were continuously measured.

Control Condition. Similar to the time and locomotor pattern of HIIT, subjects underwent a control (CON) task on the treadmill. A comfortable normal walking speed below $50 \%$ of $\mathrm{HR}_{\max }$ was adjusted for both groups for $4 \times 4 \mathrm{~min}$. During the 3 -min breaks, participants stood still in an upright position. Heart rate and gas exchange data were also collected.

\section{Testing Procedure}

Standing Balance. In order to become accustomed to the balance testing procedure, it was allowed to try all relevant standing balance tasks (twice, $10 \mathrm{~s}$ for each condition) prior to baseline recordings. A short post-familiarization break of about 2 min was completed. In order to measure static standing balance performance, a piezoelectric Kistler ${ }^{\circledR}$ force plate (KIS type 9286BA; Kistler Instruments AG, Winterthur, Switzerland) was employed and installed on an even and rigid laboratory floor. Laboratory light $(\sim 500 \mathrm{~lx})$ and temperature $\left(21^{\circ} \mathrm{C}\right)$ were kept constant. Double limb stance with eyes closed (DLEC) and single limb stance with eyes open (SLEO) were applied as balance tasks. The lateral preference inventory was employed to determine the dominant leg for SLEO testing. The first task was randomly assigned but kept constant within each individual. The tasks were then measured alternately until three attempts for each standing balance task were completed. Both standing balance tasks were tested for $10 \mathrm{~s}$. The participants were instructed to (a) perform without shoes, (b) place their feet comfortably, nearly parallel at approximately shoulder width 
Table 2. Acute cardiopulmonary responses to the HIIT and control condition (mean \pm SD values)

\begin{tabular}{|c|c|c|c|c|c|c|c|c|}
\hline & \multicolumn{4}{|c|}{ Young adults } & \multicolumn{4}{|l|}{ Seniors } \\
\hline & INT 1 & INT 2 & INT 3 & INT 4 & INT 1 & INT 2 & INT 3 & INT 4 \\
\hline \multicolumn{9}{|l|}{ HIIT } \\
\hline $\mathrm{VO}_{2}, \mathrm{ml} \cdot \mathrm{min}^{-1} \cdot \mathrm{kg}^{-1}$ & $44.5 \pm 3.5$ & $43.5 \pm 4.9$ & $44.8 \pm 4.5$ & $42.5 \pm 6.2$ & $32.0 \pm 5.7$ & $31.5 \pm 5.3$ & $32.5 \pm 3.5$ & $33.0 \pm 3.8$ \\
\hline$\% \mathrm{VO}_{2 \max }, \%$ & $93 \pm 6$ & $92 \pm 8$ & $93 \pm 7$ & $92 \pm 9$ & $96 \pm 9$ & $95 \pm 8$ & $97 \pm 6$ & $98 \pm 7$ \\
\hline $\mathrm{HR}, \min ^{-1}$ & $186 \pm 2$ & $185 \pm 5$ & $183 \pm 5$ & $186 \pm 6$ & $145 \pm 7$ & $141 \pm 7$ & $149 \pm 9$ & $151 \pm 8$ \\
\hline$\% \mathrm{HR}_{\max }, \%$ & $96 \pm 5$ & $95 \pm 2$ & $94 \pm 1$ & $95 \pm 1$ & $92 \pm 4$ & $93 \pm 4$ & $93 \pm 3$ & $94 \pm 3$ \\
\hline $\mathrm{BR}, \mathrm{min}^{-1}$ & $43 \pm 6$ & $45 \pm 7$ & $48 \pm 3$ & $45 \pm 5$ & $39 \pm 8$ & $38 \pm 6$ & $39 \pm 6$ & $38 \pm 6$ \\
\hline RER & $1.03 \pm 0.12$ & $1.04 \pm 0.14$ & $1.08 \pm 0.10$ & $1.06 \pm 0.10$ & $1.03 \pm 0.14$ & $1.04 \pm 0.22$ & $1.03 \pm 0.16$ & $0.99 \pm 0.19$ \\
\hline \multicolumn{9}{|l|}{ Control } \\
\hline $\mathrm{VO}_{2}, \mathrm{ml} \cdot \mathrm{min}^{-1} \cdot \mathrm{kg}^{-1}$ & $12.5 \pm 2.1$ & $14.5 \pm 3.5$ & $14.0 \pm 2.4$ & $13.0 \pm 2.4$ & $14.0 \pm 2.8$ & $12.5 \pm 1.7$ & $13.5 \pm 2.1$ & $15.0 \pm 2.8$ \\
\hline$\% \mathrm{VO}_{2 \max }, \%$ & $27 \pm 4$ & $30 \pm 3$ & $30 \pm 4$ & $28 \pm 4$ & $41 \pm 8$ & $39 \pm 4$ & $40 \pm 6$ & $42 \pm 8$ \\
\hline $\mathrm{HR}, \mathrm{min}^{-1}$ & $89 \pm 5$ & $91 \pm 5$ & $90 \pm 7$ & $88 \pm 8$ & $83 \pm 3$ & $82 \pm 3$ & $82 \pm 2$ & $86 \pm 3$ \\
\hline$\% \mathrm{HR}_{\max }, \%$ & $50 \pm 4$ & $49 \pm 4$ & $49 \pm 5$ & $49 \pm 5$ & $52 \pm 3$ & $50 \pm 2$ & $52 \pm 2$ & $51 \pm 4$ \\
\hline $\mathrm{BR}, \mathrm{min}^{-1}$ & $20 \pm 6$ & $21 \pm 2$ & $24 \pm 7$ & $22 \pm 6$ & $23 \pm 3$ & $22 \pm 2$ & $21 \pm 2$ & $22 \pm 2$ \\
\hline RER & $0.88 \pm 0.15$ & $0.89 \pm 0.18$ & $0.87 \pm 0.16$ & $0.88 \pm 0.19$ & $0.87 \pm 0.09$ & $0.89 \pm 0.12$ & $0.88 \pm 0.14$ & $0.90 \pm 0.14$ \\
\hline
\end{tabular}

on the platform, (c) place their hands akimbo, (d) bend the knees slightly and (e) stand as still as possible while focusing a marked circle (except during DLEC) at the nearby wall (distance $1.5 \mathrm{~m}$, height $1.75 \mathrm{~m}$ ).

Muscle Activity. Muscle activity of four lower limb muscles (mm. soleus, SOL; medial head of gastrocnemius, GM; tibialis anterior, TIB; peroneus longus, PL) was assessed according to the European recommendations for surface electromyography (SEMG) [22]. In order to achieve the required skin conductance level of $<5 \mathrm{k} \Omega$ (assessed before and after testing), the skin of the dominant leg was prepared with shavers and fine sandpaper prior to testing. Bipolar electrodes with a surface area of $1.0 \mathrm{~cm}$ and a between-electrode (Blue Sensor; Ambu A/S, Balerup, Denmark) distance of $2.0 \mathrm{~cm}$ were placed on the required spot marks while seating.

\section{Data Acquisition and Analyses}

Standing Balance. Standing balance data during SLEO and DLEC were collected within a time frame of $10 \mathrm{~s}$ using a sampling rate of $40 \times \mathrm{s}^{-1}$ [23]. A Butterworth filter with a low-pass cut-off frequency of $10 \mathrm{~Hz}$ was applied to the time signals of the ground reaction force data in order to reduce high-frequency noise contamination. Total center of pressure (COP) path length displacement served as outcome measure $[24,25]$. The mean of the path lengths of the three attempts was calculated and included into further statistical analyses.

Muscle Activity. Analogue-to-digital conversion of SEMG signals was carried out at a rate of $1,000 \times \mathrm{s}^{-1}$ using a 16-bit system (IMAGO; pfitec, Endingen, Germany). Raw SEMG data were processed offline using custom-made algorithms in MATLAB (The MathWorks, Natick, Mass., USA). After correcting the trend and removing $50 \mathrm{~Hz}$ and odd-numbered harmonics of the signals, filters with a band-pass of $20-400 \mathrm{~Hz}$ were applied [26]. Electrical activity was calculated with a moving root mean square (RMS) window of $0.2 \mathrm{~s}$ (i.e. 200 data points) and 50\% window overlap and was averaged over all segments per subject and muscle, respectively. The results were averaged over the three trials.
In order to normalize the changes, the averaged results of absolute amplitudes and RMS were normalized according to baseline values (i.e. before intervention). Values around zero would reflect unchanged activation related to baseline measurements. A RMS\% of 20 , in contrast, would represent an elevated muscle activity by $20 \%$.

\section{Gas Exchange and Heart Rate Data}

Gas exchange data were collected using a breath-by-breath spiroergometric system (Metalyzer 3B; Cortex Biophysik GmbH) and averaged for 10-s intervals. The mean of the three highest consecutive $\mathrm{VO}_{2}$ and RER values within a $30 \mathrm{~s}$ time frame of the final exercise step during the maximal exercise test was regarded as $\mathrm{VO}_{2 \text { max }}$. The highest recorded heart rate (Polar M62; Polar Electro, Oy) was regarded as $\mathrm{HR}_{\max }$. Ratings of perceived exertion (RPE, CR-10 scale) were requested at the end of each exercise step and at exhaustion. The cardiopulmonary response to HIIT and CON is provided in table 2 .

\section{Statistical Analysis}

Data analyses were carried out with Statistica 10.0 (StatSoft, Inc., Tulsa, Okla., USA). Data are provided as means with SD or 95\% CI, respectively. COP path length displacements for both stance conditions (DLEO and SLEO) were separately analyzed for both groups by means of 2 (dependent factor: 'condition'; HIIT, CON) $\times 5$ (repeating factor: 'time'; pre, post, 10 min post, $30 \mathrm{~min}$ post, $45 \mathrm{~min}$ post) repeated measures analyses of variance (rANOVAs). Fisher's least significance difference (LSD) post hoc tests were computed in case of significant main or interaction effects. In order to analyze SEMG data, we accordingly calculated several 2 (condition: HIIT vs. CON) $\times 4$ (repeating factor 'time': post, $10 \mathrm{~min}$ post, $30 \mathrm{~min}$ post, $45 \mathrm{~min}$ post) rANOVAs for each muscle (GM, SOL, TIB, PL) and stance conditions (DLEC, SLEO) separately for each age group. Thereby, effect sizes (partial eta squared, $\eta_{\mathrm{p}}{ }^{2}$ ) for the rANOVA interaction effects were calculated to estimate practical relevance. According to Cohen [27], a $\eta_{\mathrm{p}}{ }^{2}$ $\geq 0.01$ indicates a small, $\geq 0.06$ a medium, and $\geq 0.14$ a large effect.
18

Gerontology 2015;61:15-23 DOI: $10.1159 / 000363767$
Donath/Kurz/Roth/Hanssen/ Schmidt-Trucksäss/Zahner/Faude 


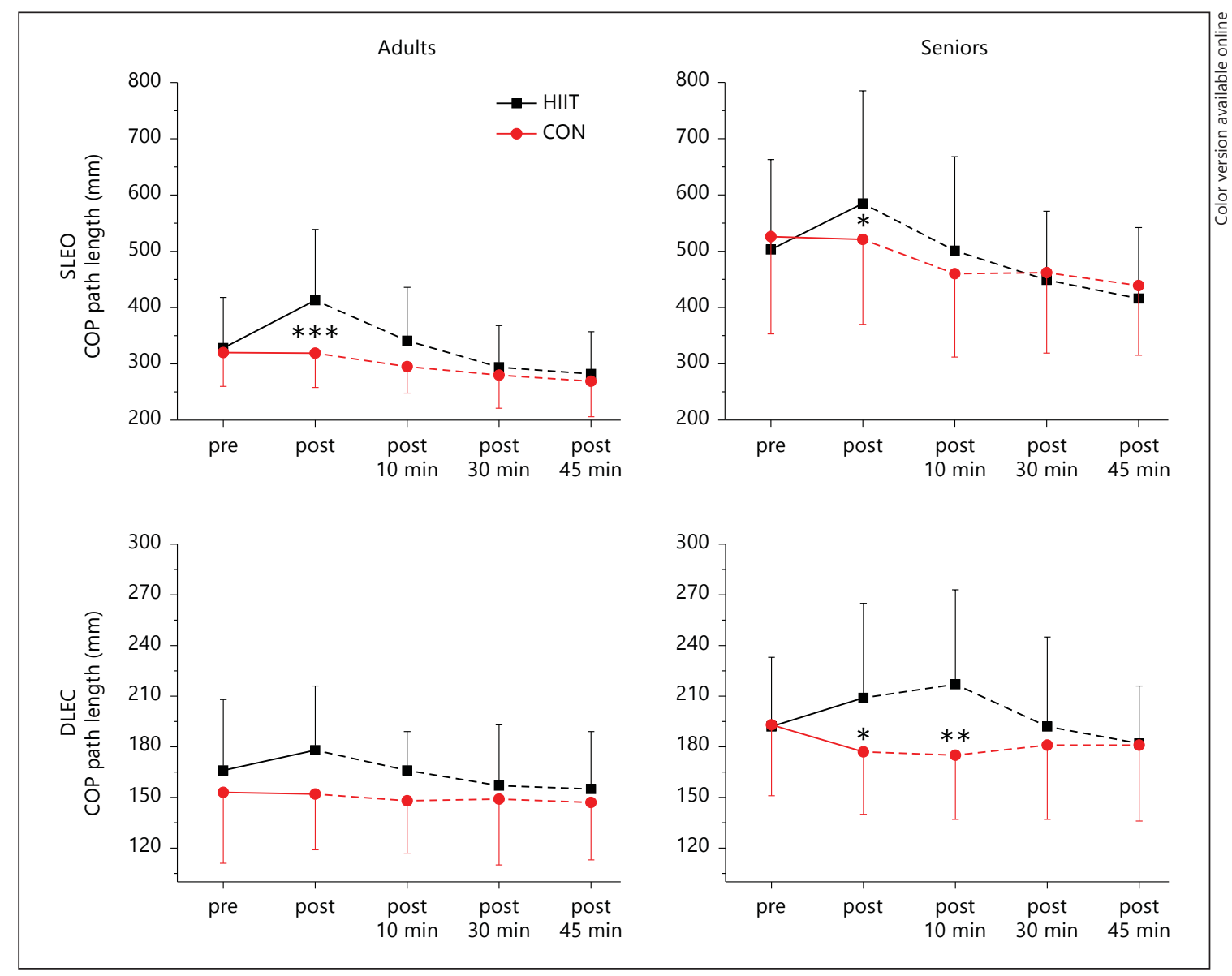

Fig. 2. COP path length displacement for young adults and seniors during SLEO and DLEC. Each subject randomly performed an acute HIIT (squares) and a CON (circles) with exercise-related (solid lines) and recoveryrelated (dashed lines) data points. Data are presented as means and SDs. ${ }^{* * *} \mathrm{p}<0.001 ;{ }^{* *} \mathrm{p}<0.01{ }^{*} \mathrm{p}<0.05$.

\section{Results}

\section{Postural Sway}

Large condition (HIIT vs. CON) $\times$ time (pre, immediately after exercise cessation, $10 \mathrm{~min}$ post, $30 \mathrm{~min}$ post, 45 min post) interactions were found for SLEO in young adults $\left(\mathrm{p}<0.001, \eta_{\mathrm{p}}{ }^{2}=0.31\right)$ and seniors $\left(\mathrm{p}=0.02, \eta_{\mathrm{p}}{ }^{2}=\right.$ 0.18 ) as well. For both age groups, COP path length displacements were increased immediately after exercise cessation (post-hoc: adults, $\mathrm{p}<0.001$; seniors, $\mathrm{p}=0.01$ ) and 10 min post (post-hoc: adults, $\mathrm{p}=0.07$; seniors, $\mathrm{p}=$ 0.08) (fig. 2).

No condition $\times$ time interaction $\left(\mathrm{p}=0.57, \eta_{\mathrm{p}}{ }^{2}=0.05\right)$ was found for DLEC in young adults. A large condition $x$ time interaction $\left(\mathrm{p}=0.04, \eta_{\mathrm{p}}{ }^{2}=0.16\right)$ was observed for DLEC in seniors. Post hoc testing for seniors revealed a difference between HIIT and CON immediately after exercise cessation $(\mathrm{p}=0.03)$ and $10 \mathrm{~min}$ post $(\mathrm{p}=0.004)$ (fig. 2$)$.

\section{Muscle Activity}

DLEC neither revealed condition (HIIT vs. CON) $\times$ time (post, 10 min post, 30 min post, $45 \mathrm{~min}$ post) interaction effects (adults: $0.44<\mathrm{p}<0.98$; seniors: $0.49<\mathrm{p}<$ 0.93 ), time effects (adults: $0.22<\mathrm{p}<0.88$; seniors: $0.31<$ $\mathrm{p}<0.77$ ) nor condition effects (adults: $0.30<\mathrm{p}<0.45$; seniors: $0.35<p<0.91$ ) for the four muscles and both groups (data not presented).

In contrast, large condition $\times$ time interaction effects were found during SLEO for TIB in adults ( $\mathrm{p}<$ $\left.0.001, \eta_{\mathrm{p}}{ }^{2}<0.33\right)$ and seniors $\left(\mathrm{p}=0.035, \eta_{\mathrm{p}}{ }^{2}<0.19\right)$ as well (fig. 3). Post hoc testing revealed differences between HIIT and CON immediately after HIIT cessation for adults $(\mathrm{p}=0.007)$ and seniors $(\mathrm{p}=0.009)$ as well as $10 \mathrm{~min}$ after exercise cessation for seniors $(\mathrm{p}=0.01)$ only (fig. 3). Condition effects were found for SOL ( $\mathrm{p}=$ $\left.0.007, \eta_{\mathrm{p}}{ }^{2}<0.50\right)$ and PL $\left(\mathrm{p}=0.004, \eta_{\mathrm{p}}^{2}<0.33\right)$ only in adults. 

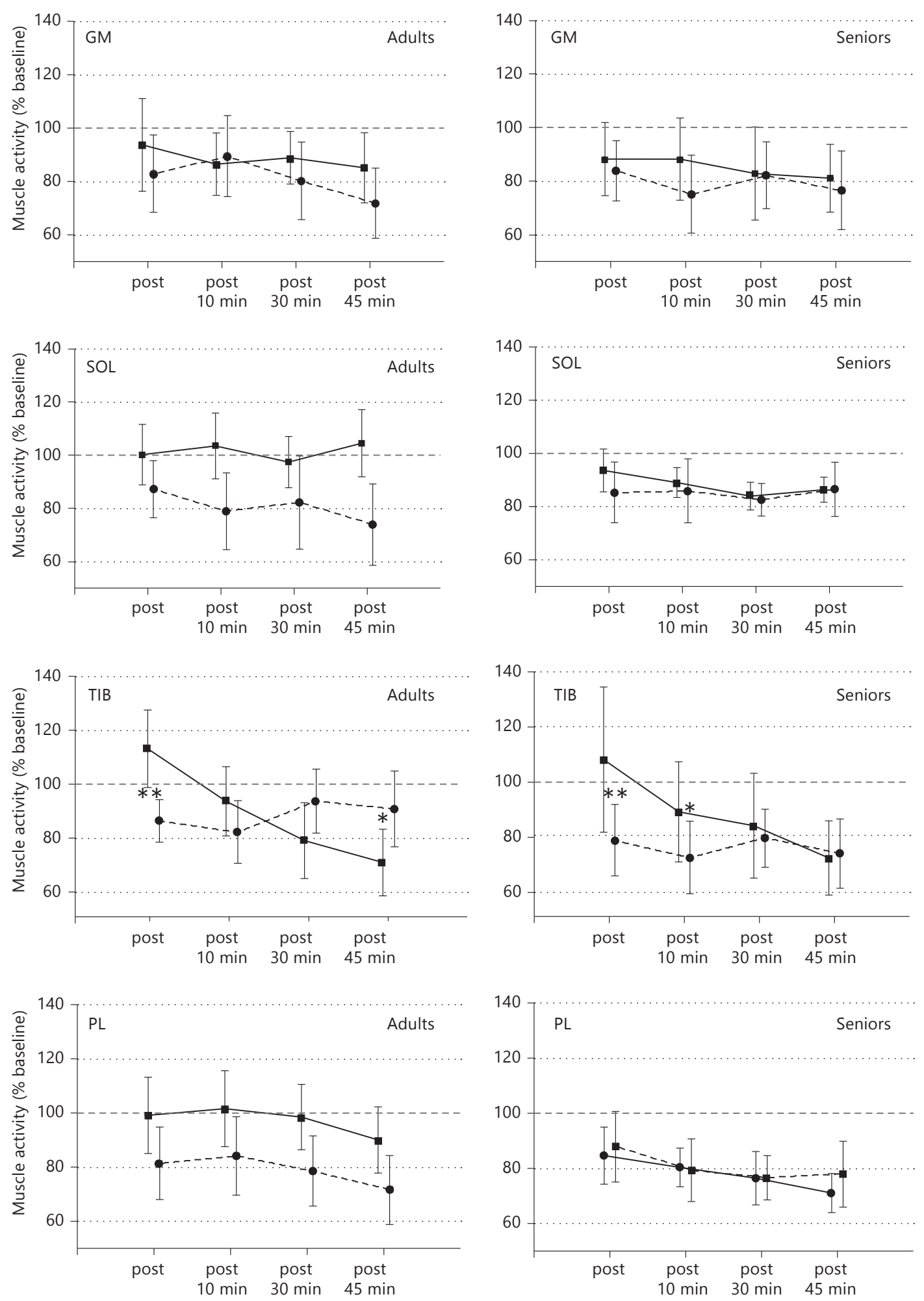

Fig. 3. Muscle activity data for the GM, SOL, TIB and PL during SLEO and DLEC quiet stance for young adults (left charts) and seniors (right charts). Data were normalized for pre-exercise baseline muscle activity (zero line) and indicate the percentage difference to baseline activity. Data are provided as means and $95 \%$ CI. ${ }^{* *} \mathrm{p}<0.01 ;{ }^{*} \mathrm{p}<0.05$. 


\section{Discussion}

The present study investigated the time course of standing balance performance and lower leg muscle activity before and after one typical bout of $4 \times 4 \mathrm{~min}$ HIIT separately for healthy young adults and seniors. Postural sway was adversely affected immediately after exercise cessation in both groups during SLEO and in seniors also during DLEC. Postural control during SLEO nearly returned to baseline in both groups within about $10 \mathrm{~min}$ of balance recovery after exercise cessation. Only seniors needed 10-30 min after DLEC until balance performance returned to baseline levels. Increased muscle activity compared to CON was only observed for TIB immediately after exercise cessation in both groups and in seniors until 10 min after HIIT.

Deteriorated postural sway immediately after HIIT during SLEO in both groups could be mainly caused by an interfered alteration of sensorimotor function and ventilation. It seems likely that the mechanical and physiological demands of high-intensity exercise adversely affect proprioception: for example, higher eccentric loads during exercise have been reported to deteriorate mechanoreceptor function [28] and may overstimulate otolith function $[29,30]$. As elevated respiration rates [31] and an enhanced discharge rate of the circulatory system [32] have been reported to impair postural control, exhaustive cardiorespiratory efforts most likely diminish postural control after intense aerobic exercise.

Standing balance performance during DLEC revealed a different picture for both groups. Whereas HIIT did not notably affect postural control during DLEC in young adults, seniors showed a prolonged deterioration of standing balance performance up to $30 \mathrm{~min}$ after exercise cessation. It has been previously shown that vision-based sensory function prevails compared to mechanoreceptor function in postural control in seniors [33]. In addition to an aging-induced decline of mechanoreceptor function in seniors [28], deprived vision may decrease overall sensory integrity with adverse effects on postural control, particularly after intense exercise. Corroboratively, comparable changes were also found after a single walkingbased treadmill test until objective exhaustion in seniors [14].

Concerning electromyographic activity, a single HIIT session altered TIB activation in both groups during SLEO, particularly pronounced immediately after exercise cessation. While altered TIB muscle activity during SLEO nearly recovered within $10 \mathrm{~min}$ in adults, the acute

Single Session of High-Intensity Interval Training effect of a single HIIT session lasted up to half an hour in the elderly. This finding might reflect a comparatively faster tibial neuromuscular balance recovery after intense exercise in adults compared to seniors. In this regard, increased ankle muscle amplitudes might not only compensate for structural and functional declines of muscle strength during aging $[34,35]$ but also for acute exerciseinduced changes of the postural control system.

In a closed kinetic chain, TIB can cause an anterior tibial translation. Thus, TIB acts antagonistically to the hamstrings [36]. TIB may counteract a backward movement of the COP during upright stance. It is, however, not clear whether an altered muscle activity after HIIT reflect an adapted strategy of the sensory-motor system to maintain balance while standing or a compensating mechanism for reduced capacities of the integrative function of the neuromuscular-skeletal system in general. Further studies need to address this issue with certainty. Although coactivation was not a primary outcome measure in this investigation, we assume that the constantly elevated muscle amplitudes of TIB in seniors during SLEO led to enhanced coactivation of the ankle muscles [37]. Coactivation has been shown to be associated with increased postural sway during standing balance tasks [38]. In this regard, an increased coactivation would stiffen the joint in order to reduce destabilization effects and thus reflect a more inefficient neuromuscular strategy.

The present study comprises some limitations that should be addressed. As physical activity has been shown to be positively associated with decreased postural sway [39], the examined seniors do not reflect a fall-prone population. It seems reasonable to assume that older and frailer seniors with additional cardiovascular comorbidities may show larger alteration of postural sway and muscle activity. Moreover, the treadmill exercise patterns during HIIT differed between both groups. Whereas seniors performed a walking-based protocol, adults followed a running-based exercise protocol. Thus, we cannot rule out with certainty whether the observed differences in muscle activity and impairments of postural control might be caused by potential differences of the underlying motor patterns between walking and running. As mechanoreceptor function seems to be likely more deteriorated after running compared to walking, the balance performance alteration in our group of older participants could be underestimated in comparison to the young adults. However, we controlled for cardioventilatory response which was similar in both groups. 


\section{Conclusion}

A single HIIT session led to a transient deterioration of postural sway during SLEO in adults and seniors. Within 10 min after HIIT cessation however, balance performance returned to baseline in both groups. HIIT mainly led to altered TIB muscle activity, particularly pronounced immediately after intervention in both groups and up to $30 \mathrm{~min}$ after exercise cessation in seniors. Postural sway during DLEC and tibial muscle activity during SLEO following a single HIIT session remained affected up to $30 \mathrm{~min}$ after exercise cessation. This potential 'open-fall-window' can lead to a higher risk to sustain falls due to deteriorated postural control, particularly in seniors. Since seniors should pay notable attention to demanding balance requirements immediately after an intense bout of physical exercise (e.g. during showering or changing clothes), the advantages of HIIT with regard to time efficiency are debatable.

It would be interesting for future studies to include kinematic analysis of the trunk and extremities in order to address whether acute exercise and exercise-induced fatigue may affect the strategy of postural control in seniors (hip vs. ankle strategy). It seems furthermore interesting whether exercise-induced fatigue processes lead to delayed muscle activity onsets and ground reaction forces when tripping incidences would be induced. Also, studies in more inactive, frail and fall-prone seniors are needed in order to disentangle whether acute exercise affects relevant intrinsic fall-risk factors. This is of more importance, since older, frailer and fall-prone seniors are unlikely to be able or willing to safely participate in the intensity of interval training described. Thus, it would be interesting for future studies to attempt to quantify the exercise dosage at which these balance perturbations become evident in older and frailer seniors. Since postural control in seniors is mainly based on the visual sensory function, balance exercise with occluded vision might be included in training regimens to enhance mechanoreceptor function. Regarding training studies, it would also be interesting to see if there is a training or adaptation effect with repeated sessions of HIIT. The present study only evaluated a single HIIT session. It might be reasonable to assume that repeatedly conducted HIIT sessions lead to adapted acute responses of balance performance.

\section{Acknowledgments}

The authors appreciate the engagement of the subjects during the study. Additional thanks are dedicated to Tobias van Baarsen and Claudia Egli (Basel, Switzerland) who completed exercise testing and collected balance and muscle activity data.

\section{Disclosure Statement}

The authors have no conflicts of interest to disclose.

\section{References}

1 Lutz W, Sanderson W, Scherbov S: The coming acceleration of global population ageing. Nature 2008;451:716-719.

$\checkmark 2$ Gandjour A: Healthcare expenditures from living longer-how much do they matter. Int $J$ Health Plann Manage (Epub ahead of print).

$\checkmark 3$ Rubenstein LZ: Falls in older people: epidemiology, risk factors and strategies for prevention. Age Ageing 2006;35(suppl 2):ii37ii41.

-4 Stevens JA, Corso PS, Finkelstein EA, Miller TR: The costs of fatal and non-fatal falls among older adults. Inj Prev 2006;12:290295.

5 Hauer K, Becker C, Lindemann U, Beyer N: Effectiveness of physical training on motor performance and fall prevention in cognitively impaired older persons: a systematic review. Am J Phys Med Rehabil 2006;85: 847-857.

-6 Trombetti A, Hars M, Herrmann FR, Kressig RW, Ferrari S, Rizzoli R: Effect of music- based multitask training on gait, balance, and fall risk in elderly people: a randomized controlled trial. Arch Intern Med 2011;171:525533.

-7 Gillespie LD, Robertson MC, Gillespie WJ, Sherrington C, Gates S, Clemson LM, Lamb SE: Interventions for preventing falls in older people living in the community. Cochrane Database Syst Rev 2012;9:CD007146.

-8 Granacher U, Muehlbauer T, Zahner L, Gollhofer A, Kressig RW: Comparison of traditional and recent approaches in the promotion of balance and strength in older adults. Sports Med 2011;41:377-400.

-9 Helbostad JL, Leirfall S, Moe-Nilssen R, Sletvold $\mathrm{O}$ : Physical fatigue affects gait characteristics in older persons. J Gerontol A Biol Sci Med Sci 2007;62:1010-1015

10 Helbostad JL, Sturnieks DL, Menant J, Delbaere K, Lord SR, Pijnappels M: Consequences of lower extremity and trunk muscle fatigue on balance and functional tasks in older peo- ple: a systematic literature review. BMC Geriatr 2010;10:56.

11 Parijat P, Lockhart TE: Effects of lower extremity muscle fatigue on the outcomes of slip-induced falls. Ergonomics 2008;51:18731884.

12 Granacher U, Gruber M, Forderer D, Strass D, Gollhofer A: Effects of ankle fatigue on functional reflex activity during gait perturbations in young and elderly men. Gait Posture 2010;32:107-112.

13 Stemplewski R, Maciaszek J, Salamon A, Tomczak M, Osinski W: Effect of moderate physical exercise on postural control among 65-74 year old men. Arch Gerontol Geriatr 2012;54:e279-e283.

14 Donath L, Zahner L, Roth R, Fricker L, Cordes M, Hanssen H, Schmidt-Trucksass A, Faude $\mathrm{O}$ : Balance and gait performance after maximal and submaximal endurance exercise in seniors: is there a higher fall-risk? Eur J Appl Physiol 2013;113:661-669. 
$\checkmark 15$ Helgerud J, Hoydal K, Wang E, Karlsen T, Berg P, Bjerkaas M, Simonsen T, Helgesen C, Hjorth N, Bach R, Hoff J: Aerobic high-intensity intervals improve $\mathrm{VO}_{2 \max }$ more than moderate training. Med Sci Sports Exerc 2007;39:665-671.

-16 Molmen HE, Wisloff U, Aamot IL, Stoylen A, Ingul CB: Aerobic interval training compensates age-related decline in cardiac function. Scand Cardiovascr J 2012;46:163-171.

$\checkmark 17$ Wisloff U, Stoylen A, Loennechen JP, Bruvold M, Rognmo O, Haram PM, Tjonna AE, Helgerud J, Slordahl SA, Lee SJ, Videm V, Bye A, Smith GL, Najjar SM, Ellingsen O, Skjaerpe T: Superior cardiovascular effect of aerobic interval training versus moderate continuous training in heart failure patients: a randomized study. Circulation 2007;115:3086-3094.

- 18 Nemoto K, Gen-no H, Masuki S, Okazaki K, Nose H: Effects of high-intensity interval walking training on physical fitness and blood pressure in middle-aged and older people. Mayo Clin Proc 2007;82:803-811.

19 Frey I, Berg A, Keul J: Assessment and evaluation of leisure activity of participants in heart rehabilitation groups. Wien Klin Wochenschr 1995;107:774-777.

20 Peterson MJ, Pieper CF, Morey MC: Accuracy of $\mathrm{VO}_{2 \max }$ prediction equations in older adults. Med Sci Sports Exerc 2003;35:145149.

21 Midgley AW, McNaughton LR, Polman R, Marchant D: Criteria for determination of maximal oxygen uptake: a brief critique and recommendations for future research. Sports Med 2007;37:1019-1028.

-22 Hermens HJ, Freriks B, Disselhorst-Klug C, Rau G: Development of recommendations for SEMG sensors and sensor placement procedures. J Electromyogr Kinesiol 2000;10:361374.
Donath L, Roth R, Zahner L, Faude O: Testing single and double limb standing balance performance: comparison of COP path length evaluation between two devices. Gait Posture 2012;36:439-443.

-24 Clark RA, Bryant AL, Pua Y, McCrory P, Bennell K, Hunt M: Validity and reliability of the Nintendo Wii Balance Board for assessment of standing balance. Gait Posture 2010;31: 307-310.

25 Salavati M, Hadian MR, Mazaheri M, Negahban H, Ebrahimi I, Talebian S, Jafari AH, Sanjari MA, Sohani SM, Parnianpour M: Test-retest reliability (corrected) of center of pressure measures of postural stability during quiet standing in a group with musculoskeletal disorders consisting of low back pain, anterior cruciate ligament injury and functional ankle instability. Gait Posture 2009;29:460-464.

26 Kurz E, Herbsleb M, Anders C, Puta C, Vollandt R, Czepa D, Ziezio R, Scholle HC, Hilberg T: SEMG activation patterns of thigh muscles during upright standing in haemophilic patients. Haemophilia 2011;17:669-675.

27 Cohen J: Statistical Power Analysis for the Behavior Sciences, ed 2. New York, Erlbaum Associates, 1988.

28 Riskowski JL, Mikesky AE, Bahamonde RE, Alvey TV 3rd, Burr DB: Proprioception, gait kinematics, and rate of loading during walking: are they related? J Musculoskelet Neuronal Interact 2005;5:379-387.

29 Lepers R, Bigard AX, Diard JP, Gouteyron JF, Guezennec CY: Posture control after prolonged exercise. Eur J Appl Physiol Occup Physiol 1997;76:55-61.

30 Charles C, Cian C, Nougier V, Bigard XA, Job A, Raphel C: Overstimulation of the vestibular system and body balance. J Vestib Res 2002;12:135-143.

- 31 Schmid M, Conforto S, Bibbo D, D’Alessio T: Respiration and postural sway: detection of phase synchronizations and interactions. Hum Mov Sci 2004;23:105-119.
32 Caron O, Fontanari P, Cremieux J, Joulia F: Effects of ventilation on body sway during human standing. Neurosci Lett 2004;366:6-9.

33 Colledge NR, Cantley P, Peaston I, Brash H, Lewis S, Wilson JA: Ageing and balance: the measurement of spontaneous sway by posturography. Gerontology 1994;40:273-278.

34 Pijnappels M, Reeves ND, Maganaris CN, van Dieen JH: Tripping without falling; lower limb strength, a limitation for balance recovery and a target for training in the elderly. J Electromyogr Kinesiol 2008;18:188-196.

35 Horlings CG, Kung UM, van Engelen BG, Voermans NC, Hengstman GJ, van der Kooi AJ, Bloem BR, Allum JH: Balance control in patients with distal versus proximal muscle weakness. Neuroscience 2009;164:1876-1886.

36 Elias JJ, Faust AF, Chu YH, Chao EY, Cosgarea $\mathrm{AJ}$ : The soleus muscle acts as an agonist for the anterior cruciate ligament. An in vitro experimental study. Am J Sports Med 2003; 31:241-246.

37 Nagai K, Yamada M, Tanaka B, Uemura K, Mori S, Aoyama T, Ichihashi N, Tsuboyama $\mathrm{T}$ : Effects of balance training on muscle coactivation during postural control in older adults: a randomized controlled trial. J Gerontol A Biol Sci Med Sci 2012;67:882-889.

38 Nagai K, Yamada M, Uemura K, Yamada Y, Ichihashi N, Tsuboyama T: Differences in muscle coactivation during postural control between healthy older and young adults. Arch Gerontol Geriatr 2011;53:338-343.

- 39 Kiers H, van Dieen J, Dekkers H, Wittink H, Vanhees L: A systematic review of the relationship between physical activities in sports or daily life and postural sway in upright stance. Sports Med 2013;43:1171-1189.

40 Tanaka H, Monahan KD, Seals DR: Age-predicted maximal heart rate revisited. J Am Coll Cardiol 2001;37:153-156. 